\title{
Tisser le récit de sa vie avec autrui. Images de soi et jeux de mémoire dans L'Homme effacé de Michel Ouellette
}

\author{
Emir Delic \\ Université d'Ottawa
}

[L]e je n'existe pas sans un tu. On ne peut accéder au fond de soi si l'on en exclut les autres. (Tzvetan Todorov, Les Morales de l'histoire, p. 130)

On pense que c'est le passé qui fait le présent. Mais, moi, je me dis que c'est peut-être plus l'avenir. Parce qu'on a ben plus besoin de l'avenir que du passé. Dans le fond. Le présent, c'est juste une bataille entre le passé pis l'avenir. Pis c'est ben à nous autres de choisir quel bord gagne.

(Michel Ouellette, L'Homme effacé, p. 90) 
$\mathrm{Au}$ premier regard, l'expression élémentaire de l'identité d'un sujet paraît simple, voire triviale : le soi n'est pas l'autre. Tout se complique toutefois dès que l'on cherche à cerner la nature des rapports entre les deux termes de la relation. À partir du constat de leur opposition, l'on pourrait effectivement être enclin à avancer que, dans une perspective purement logique, l'altérité consiste en une simple négation de l'identité. C'est sur une telle négation que repose l'approche de l'autre proposée par Charles Renouvier: «Le sujet, écrit-il dans une note repérée par Louis Prat, ma pensée propre. L'objet : un coup du dehors. L'objet c'est l'autre»(p. 9) ${ }^{1}$. Les risques de pareilles visions objectivantes de l'autre, notamment dans le domaine des jugements de valeur, ne sont pas à sous-estimer. Les idéologies coloniales, toutes fondées sur le principe néfaste d'une inadéquation foncière entre colonisateurs et colonisés, nous l'auront appris avec force. Comme le fait valoir Albert Memmi à ce sujet,

[l']abaissement du colonisé, qui doit expliquer son dénuement, sert en même temps de repoussoir à la positivité du colonialiste. [...] Comparaisons morales ou sociologiques, esthétiques ou géographiques, explicites, insultantes ou allusives et discrètes, mais toujours en faveur de la métropole et du colonialiste. Ici, les gens d'ici, les mœurs de ce pays, sont toujours inférieurs, et de loin, en vertu d'un ordre fatal et préétabli. (p. 87)

Infailliblement, sont alors assignés à l'être génie, positivité et supériorité; au non-être, à "celui qui n'“en est" pas » (Kristeva, 2004, p.139), nullité, négativité et infériorité. On aurait tort d'ailleurs de supposer que ce genre de perspectives essentialistes et de réifications d'autrui appartien-

\footnotetext{
${ }^{1}$ Nous devons cette découverte à l'ouvrage magistral d'André Lalande (2006, p. 39).
} 
nent à l'Histoire. Que l'on songe ici à de récentes manifestations de xénophobie et de racisme $^{2}$ - les multiples génocides du $\mathrm{XX}^{\mathrm{e}}$ siècle en constituant des aboutissements parmi les plus radicaux qui soient - , qui, en s'attaquant au statut même d'un être humain, présupposent l'insuffisance de l'être de l'autre, c'est-à-dire lui attribuent un non-être. L'opposition entre le soi et l'autre nous renvoie ainsi au point névralgique de la problématique des rapports entre deux entités.

De toute évidence, du fait même qu'il institue son identité sur la base de traits différentiels qui fondent son unicité, le soi écarte l'autre, mais il faudrait éviter le piège qui consiste à assimiler cette mise à l'écart à une relation privative. Dans une étude des processus d'exclusion qui fait appel aux concepts foucaldiens de raison et de déraison, Machiel Karskens nous livre des remarques instructives à ce sujet ${ }^{3}$ :

By definition, the notions reason and unreason are mutually exclusive and irreconcilable. For unreason is defined as the absence or privation of reason, nothing more. [...] According to that logic [the logic of privation], only the positive term can provide the relationship with content. The positive term must be a property or quality that the subject (of predication) ought to have, but does not necessarily possess. Furthermore, there is no middle way between the presence or absence of that property. In the case of privation as distinguished from other types of negation, the property must be a real, natural or intrinsic characteristic of the subject under consideration. (p. 78-79)

L'absence ou la privation de raison - quel que soit son contenu spécifique - signifie donc inéluctablement déraison.

\footnotetext{
2 Pour une discussion intéressante des questions de «nation » et de « race » en lien avec les relations avec l'autre, voir Tzvetan Todorov (2001).

3 Karskens se réfère à Michel Foucault (1972) (1991, p. 75).
} 
Pourtant, ce genre de relation privative ne caractérise pas tous les rapports oppositionnels. Le vérifie, pour reprendre un exemple cité par Karskens, le cas de la cécité. Une personne aveugle, en effet, n'est pas simplement une personne dépourvue de la faculté de la vue étant donné qu'elle possède d'ordinaire un éventail d'aptitudes développées justement à cause de sa situation particulière. Ces traits positifs, y compris, entre autres, un aiguisement des facultés auditive et olfactive, seraient évacués d'une description appropriée de la personne aveugle si la cécité était tenue pour rien de plus qu'une absence de la vue4. Les rapports entre vue et cécité s'avèrent, au fond, analogues à ceux entre identité et altérité. En ce sens, si le soi s'oppose à autrui, leur relation ne se laisse pas non plus ramener à une privation ${ }^{5}$. L'altérité ne saurait donc être une pure absence d'identité, une non-identité, mais une identité autre. Bref, le soi n'est pas l'autre, mais ce dernier est; il est simplement autrement.

Or, tout en reposant sur une différentiation ou une séparation, le processus d'individualisation implique également un rapprochement inéluctable. C'est que les identités respectives du soi et de l'autre se modèlent, se maintiennent et

\footnotetext{
4 Voir Karskens, 1991, p. 79-80.

${ }^{5}$ Nous ne suivons pas jusqu'au bout l'argumentaire de Karskens. D’après lui, « $[\mathrm{w}]$ hen identity is seen as a very special and unique quality possessed only by special kinds of things -- e.g. man in contrast with beast, or man as opposed to woman [...], or people with identity cards in contrast to gypsies or refugees -- then it is possible to imagine alterity as the absence of identity. » (p. 82) Bien que Karskens prenne soin d'insister sur la particularité de ces cas, on ne peut, dans l'optique de la présente étude qui s'attache aux relations humaines, admettre cette possibilité. Sinon, ce serait nier aux femmes, aux habitants sans papiers comme à tout autre groupe ayant des traits différentiels qui fondent justement leur unicité, une identité qui leur est propre.
} 
évoluent dans une relation intersignificative, hautement dialogique, à telle enseigne que nous pouvons affirmer, à l'instar de Mikhaïl Bakhtine, qu' " [ê]tre signifie communiquer » (1979, p. 312; cité par Todorov, 1981, p. 148). Loin d'être de facture privative et de ménager par là la possibilité d'un quelconque solipsisme, les rapports qu'entretient le soi avec l'autre se caractérisent donc au contraire par une interdépendance fondamentale.

Le propos de la présente étude est d'explorer cette «communication profonde» (ibid.) qui détermine les identités du soi et de l'autre sous un angle particulier, à savoir celui de la mémoire. Elle vise plus précisément à examiner, à travers une réflexion sur L'Homme effacé de Michel Ouellette, la place qu'occupe l'autre dans les reconfigurations mémorielles à l'œuvre dans les continuités et les ruptures identitaires. Pour commencer, il s'agira d'esquisser à grands traits les liens qui unissent le soi à autrui au sein de l'expérience mémorielle. Ensuite, sera sondée la manière dont l'autre intervient dans les reconfigurations de la mémoire avant que soit abordé, à la fin, le rôle de la réflexivité dans le façonnement continuel des images de soi.

\section{Partages : la mémoire et la mise en récit de soi}

S'interroger sur la mémoire revient à s'interroger sur l'évocation d'un passé qui persiste malgré l'implacable fuite du temps. Parce que les moments recueillis par différentes formes mnémoniques, soient-ils agréables, douloureux ou les deux à la fois, constituent d'emblée les vestiges d'expériences vécues. 
C'est ce qu'exprime parfaitement Cécile Lamothe, l'un des personnages dans Tombeaux, le premier roman de Michel Ouellette, quand elle constate que «[l]a mémoire est un cimetière où se produit le miracle de la résurrection" (p.68). N'empêche que la parole mémorielle n'est pas seulement porteuse des débris de qui on était, mais aussi du tracé de qui on est, voire des traces de qui on sera.

Que la mémoire affecte l'inscription de notre être dans le temps ne veut pas dire pour autant qu'elle fournisse, comme l'a proposé John Locke (1788), l'unique assise à la construction de l'identité personnelle ${ }^{6}$. Car, alors qu'il ne fait pas de doute que la mémoire est gardienne d'une certaine pérennité et qu'elle fait, à ce titre, partie intégrante des formations identitaires, ce dont elle est dépositaire n'est nullement réductible à un substrat inaltérable. En témoignent au moins deux éléments, intimement liés l'un à l'autre, qui se trouvent impliqués dans la fabrication de l'histoire personnelle.

Tout d'abord, il y a la forme sous laquelle se manifeste le rappel d'un souvenir. Comme le souligne avec acuité Paul Ricœur dans La mémoire, l'histoire et l'oubli,

[i]l semble bien que le retour du souvenir ne puisse se faire que sur le mode du devenir-image. [...] La menace permanente de confusion entre remémoration et imagination, résultant de ce devenir-image du souvenir, affecte l'ambition de fidélité en laquelle se résume la fonction véritative de la mémoire. (p. 7)

S'actualisant dans l'obscurité de l'intervalle, entre présent et passé, entre présence et absence, entre réalité et imaginaire, le devenir-image du souvenir peut alors difficilement se dérober aux altérations auxquelles le soumet le passage du

${ }^{6}$ À ce sujet, on lira avec intérêt John Perry (dir.) (2008). 
temps. Viendront ici volontiers à l'esprit les ajouts, les suppressions ou les renchérissements caractéristiques de toute représentation du passé. Il va sans dire que ces changements dans le devenir-image du souvenir finissent par entraîner des changements divers dans l'identité, ou mieux, dans le devenirimage de soi du sujet engagé, bon gré mal gré, dans une (ré)exploration mnésique. C'est que l'image de soi, plurielle et dynamique de nature, consiste en une continuelle construction $\mathrm{du}$ sens de soi7. Elle relève donc fondamentalement d'une interprétation qui, suivant encore ici la pensée de Paul Ricœur, passe au premier chef par une mise en récit puisant autant dans l'histoire que dans la fiction :

Dire l'identité d'un individu ou d'une communauté, affirme le philosophe, c'est répondre à la question:qui a fait telle action? qui en est l'agent, l'auteur? Il est d'abord répondu à cette question en nommant quelqu'un, c'est-à-dire en le désignant par un nom propre. Mais quel est le support de la permanence du nom propre? Qu'est-ce qui justifie qu'on tienne le sujet de l'action, ainsi désigné par son nom, pour le même tout au long d'une vie qui s'étire de la naissance à la mort. La réponse ne peut être que narrative. (1991, p. 442)

Et Ricœur de préciser : «[...] [L]'histoire d'une vie ne cesse d'être refigurée par toutes les histoires véridiques ou fictives qu'un sujet raconte [et, nous ajouterions même par anticipation, fait raconter] sur lui-même » (ibid., p. 443). Aussi bien les fluctuations identitaires de tout sujet, c'est-à-dire son devenir-image de soi ou son image de soi en devenir, sont-elles déterminées par les continuelles compositions, décompositions

\footnotetext{
7 Eu égard à cette définition, l'expression « image de soi » est donc prise dans cette étude au sens du terme (bien connu, mais n'en pas moins problématique) d'identité. À la différence de celui-ci, toutefois, l'expression « image de soi » présente sans aucun doute des avantages pour ainsi dire didactiques. D’où la préférence quant à son utilisation.
} 
et recompositions des intrigues qui trament le récit d'une vie entière ${ }^{8}$.

Le second facteur clef infléchissant la fabrication de l'histoire personnelle est l'implication de l'autre aussi bien dans l'intrigue de cette histoire que dans son interprétation. À bien des égards, en effet, l'expérience mémorielle est une drôle de solitude. Elle œuvre à même le lieu d'une absence dans le dessein de conjurer une présence, présence de soi à soi-même, certes - la mémoire, on le sait, ne se passe pas d'attribution, elle est toujours mémoire $d e-$, mais aussi, voire surtout, présence de soi à l'autre - la mémoire, on le reconnaît moins volontiers, élude toute attribution univoque, elle est toujours mémoire de plusieurs. De fait, si, comme nous venons de le constater, l'image de soi ne peut être saisie qu'en (se) racontant l'histoire d'une vie, la mémoire ne constitue-t-elle pas justement le lieu originaire abritant ces différentes histoires racontées? Et, au sein de cette demeure mémorielle, autrui ne s'y anime-t-il pas diversement, tantôt en arrière-fond, tantôt en avant-scène du devenir du soi? Autrement dit, la mémoire n'estelle pas essentiellement partage? Tout porte à le croire puisque, ainsi que l'a montré en clair Wilhelm Schapp dans son ouvrage In Geschichten verstrickt, les histoires de vie, qu'elles fassent ou non l'objet d'une narration, s'entrelacent immanquablement :

\footnotetext{
8 Sur cette question, se reporter à Paul Ricœur, Soi-même comme un autre (1996), où la notion d'identité narrative, après avoir été avancée dans Temps et récit III (1991, p. 439-448), se voit pleinement développée.

9 Pierre Ouellet fait observer à ce sujet : «Quelque chose reste, qu'on voudrait bien appeler les restes mais on ne sait trop de qui ni de quoi. On marche sur des traces, mais on ne peut dire de qui elles sont, de quoi elles gardent la mémoire » (p. 161).
} 
Wir Menschen sind immer in Geschichten verstrickt. Zu jeder Geschichte gehört ein darin Verstrickter. Geschichte und InGeschichte-verstrickt-sein gehören so eng zusammen, daß man beides vielleicht nicht einmal in Gedanken trennen kann. [...]

Mit Geschichten, die uns beschäftigen, schlafen wir abends ein, sie begleiten uns und verfolgen uns bis in die Träume hinein und stehen beim Erwachen wieder neben uns. In all diesen überlieferten oder von uns selbst erlebten Geschichten gibt es den Verstrickten oder die Verstrickten, die gleichsam als Mittelpunkt die Geschichte zusammenhalten ${ }^{10}$. (p. 1)

Il s'ensuit que l'implication de l'autre dans les (re)figurations de l'histoire personnelle et dans les (re)modelages des images de soi qui en résultent va bien audelà de l'impact qu'il peut avoir dans le cadre distinctement circonscrit d'un face-à-face. Le soi ne saurait, en définitive, se comprendre, saisir qui il est, envers soi-même et envers l'autre, sans le passage obligé par cet autre, tant leurs identités narratives respectives s'entremêlent et s'influencent.

Cet enchevêtrement des histoires de vie couplé à la dialectique oubli/remémoration constitue, à n'en pas douter, une problématique qui traverse le corpus de maints écrivains de la francophonie canadienne ${ }^{11}$, mais elle est particulièrement prégnante chez l'auteur franco-ontarien Michel Ouellette. Cela

\footnotetext{
10 « Nous, les êtres humains, sommes toujours enchevêtrés dans des histoires. À chaque histoire appartient quelqu'un qui y est enchevêtré. Histoire et êtreenchevêtré-dans-une-histoire vont tellement de pair que les deux ne se laissent peut-être même pas séparer en pensée. [...] Le soir, nous nous couchons avec des histoires qui nous préoccupent; elles nous accompagnent et nous poursuivent jusqu'aux rêves et se retrouvent à nos côtés à l'éveil. Dans toutes ces histoires transmises ou vécues par nous-mêmes, il y a celui ou ceux qui s'y trouvent enchevêtrés, qui, en tant que point central pour ainsi dire, tiennent ensemble l'histoire. » Nous traduisons.

11 On songera, pour ne donner que quelques exemples, à Ying Chen, France Daigle, Abla Farhoud, Didier Leclerc, Naïm Kattan, Sergio Kokis, Marguerite-A. Primeau, Daniel Poliquin et Gabrielle Roy.
} 
dit, la question ici sera, non pas d'analyser l'évolution de la thématique de la parole mémorielle dans l'ensemble de son œuvre, mais plutôt d'examiner le traitement fort curieux que l'auteur y réserve dans sa pièce de théâtre intitulée L'Homme effacé.

\section{Images de soi en devenir}

Publiée en 1997, L'Homme effacé nous fournit un exemple d'autant plus frappant de l'entrelacement d'identités narratives que l'œuvre est centrée sur un héros muré dans un mutisme sans répit. Vêtu de "guenilles», sans «rien dans les poches » susceptible de l'identifier — «[p]as de papiers», «[p]as de numéro», «[p]as de clé », «[p]as de char», «[p]as d'adresse »-, exception faite d'un sac d'épicerie qu'il traîne obstinément avec lui, Thomas est arrêté par la police pour s'être promené en plein milieu de la rue Yonge, une artère majeure du centre-ville de Toronto (p. 22-23). Comme il refuse de parler et que les autorités «[ne] savent pas qui [il est] » (p. 21), il se voit interné dans un hôpital psychiatrique pour évaluation, en même temps qu'est publiée une annonce dans un journal afin de trouver quelqu'un capable de l'identifier. Ces efforts pour lever le voile sur l'identité du vagabond porteront leurs fruits. Annie - la femme dont il avait été amoureux dix ans plus tôt à Sudbury et qui, ne se croyant pas à l'époque en état de mettre au monde leur enfant, l'avait quitté sans préavis pour se faire avorter à Toronto - non seulement le reconnaît, mais encore se décide à lui rendre visite. En cela, elle suit, à première vue, l'avis des psychiatres selon qui «ça pourrait peut-être [lui] faire du bien de [la] revoir» (p. 24). L'intrigue 
s'ouvre sur cette première rencontre entre Thomas et Annie, laquelle ne produira pas l'effet escompté.

De fait, bien qu'Annie soit en mesure de fournir aux autorités, qui « avaient besoin de [s]on nom, de [s]on histoire » (p. 26), les renseignements demandés, Thomas gardera le silence : «PITE : Les mots restent pognés dans ta gorge. Dans ta gorge : des barreaux, des cadenas, des serrures, des chaînes. Pas de clés, pas de poignées de porte. Dans ta gorge : une prison » (p. 24).

Si Thomas s'enferme dans le silence tout au long de la pièce, c'est que le poids d'un passé douloureux pèse trop lourd sur lui. L'attestent trois personnages fantomatiques qui habitent et tourmentent sa conscience, soit Marthe, sa mère qui, atteinte d'un cancer des poumons, a commis, le jour même du départ d'Annie dix ans plus tôt, un suicide assisté avec l'aide de Pite; Pite, le locataire qui habitait la maison de la mère et qui s'imaginait une sorte de substitut de père/mari; et Annie-2, c'est-à-dire Annie à l'âge de 20 ans. Ce « trio vocal incarnant sa mémoire trouble» (Ouellette, 2007, p. 61), pour emprunter le descriptif qu'en donne l'auteur lui-même, pointe vers la source du mutisme du personnage : "MARTHE : C'est quoi les mots quand tout ce qui te reste dans la tête, c'est trois fantômes qui arrêtent pas de parler pour toi » (p. 27). En effet, la thématique de l'incommunicabilité, une constante dans l'œuvre de Michel Ouellette, se voit développée de manière fort approfondie dans cette pièce. Comme le note Jane Moss à ce propos, «Ouellette repeatedly underscores the importance of speech in L'Homme effacé by the use of the words "parler[,]" "dire," and "mots." » (p. 610) Toujours est-il que c'est seulement au tout dernier 
moment de la pièce que Thomas aura recours au pouvoir de la parole et au contact avec l'autre qu'elle assure.

Mais l'emprise des trois fantômes ne fait pas qu'entraver la communication du héros avec l'autre; elle va jusqu'à régir son existence même. En témoignent les trois objets qu'il conserve dans son sac d'épicerie, lui-même usé par le temps, à savoir un "carton de cigarettes», un «Playboy» et des «batteries" (p. 25). Ces objets, que Thomas était naguère tenu à procurer de manière régulière respectivement à Marthe, à Pite et à Annie-2, sont investis d'une charge mnémonique forte, pour ne pas dire démesurée : «MARTHE : Toute ta vie, là. Tous tes souvenirs. Ce qui reste de. Tout » (ibid.). C'est dans le but précis de faire face à ces souvenirs écrasants que Thomas essaie de s'isoler. Il n'est donc guère surprenant que, lors de sa première visite, Annie ne réussisse pas à briser le silence dans lequel il se cantonne.

Ainsi, le lendemain, tout en prétendant que c'est bien malgré elle, Annie retourne à l'hôpital psychiatrique dans l'espoir de le faire parler: "ANNIE: Ils m'ont demandé de revenir. Hier, je leur ai dit qui t'étais. Mais là, ils veulent savoir si t'as perdu la mémoire ou ben si t'es juste fou. Fa que. Ça serait bon que tu parles, hein? » (p. 33)

Dès lors, elle s'appliquera à éveiller nombre de souvenirs afin de «le franchir à deux, le silence qui [les] sépare » (p. 69). Ce processus de remémoration, initié dès le début de la pièce, s'effectue sur un mode dialogique en ce sens que l'évocation de chaque souvenir suscite une série de réactions de la part de tous les personnages -- réels et fantasmatiques --, allant du simple rappel de leur version des événements rapportés à des commentaires sur ces événements en passant, dans une veine 
presque métathéâtrale ou métacritique, par des remarques sur la situation d'interlocution :

ANNIE: Ta mère. Quand je suis arrivée chez vous avec mes affaires. Tu t'en souviens? Ta mère nous attendait. T'avais vidé mon appartement, chargé ton truck. Pis ta mère nous attendait, toute souriante, dans la cuisine.

MARTHE : Les voilà.

PITE : C'est pas une bonne affaire que tu fais là, Marthe.

MARTHE : C'est pour son bonheur.

PITE : Elle vient s'accoter avec lui dans ta maison. Ça annonce rien de bon.

ANNIE : C'était la première fois que je voyais ta mère sans son masque d'oxygène. Ça m'avait ben touchée. C'est que. On aurait dit qu'elle faisait un grand effort pour m'accueillir. Elle avait mis une belle robe. Elle s'était même maquillée.

ANNIE-2 : Le beau comité d'accueil : le vieux croque-mort pis son crown débile. Regardes-y donc la face, à celle-là. Elle est épeurante. Arrêtez de sourire de même. J'ai l'impression que vous m'attendez pour le dîner pis que c'est moi le plat principal. $[\ldots]$

ANNIE : J'y ai dit ben des affaires, à ta mère, que j'aurais pas dû lui dire.

ANNIE-2 : Maudite hypocrite!

ANNIE: Aujourd'hui, je le regrette ben. Mais on peut rien changer du passé, hein? J'étais trop jeune pour ce qui m'arrivait, pour ce qui se passait dans la maison de ta mère [sic]. (p. 39-41)

Le deuxième jour de la visite d'Annie à l'hôpital donne ainsi lieu à une série d'échanges constitués à la fois d'observations sur le présent ou le passé proche et de remises en mémoire où les propos d'Annie et ceux des trois personnages fantomatiques, en se heurtant et en se complétant, nous livrent des éclairages contrastés de souvenirs ranimés. Qu'il s'agisse des ambitions familiales du «vieux garçon » (p. 48) qu'était Pite, de la vie dure d'Annie, qui, de fille mal comprise et mal aimée, est devenue femme travailleuse et 
responsable, de Marthe, qui, sur son lit de mort, encourageait Annie à garder l'enfant qu'elle portait, ou bien de Thomas, qui se voyait tiraillé entre toutes les pressions exercées sur lui par son pseudo-père, sa mère et son amoureuse, nous en apprenons davantage sur les histoires de vie emmêlées des personnages. De la sorte, de page en page, avec une insistance toujours grandissante, ces divers fragments de récits de vie mettent en relief ce que l'expérience mnémique tend à passer sous silence : une chose est l'eccéité du moment vécu; une autre, la résurrection du moment révolu.

C'est dans cet écart entre présent et passé comme entre les affects différents qui s'y greffent que se déploient au fil du temps nombre de reconfigurations mémorielles qui, parce qu'elles opèrent souvent sournoisement, peuvent entraîner des effets tout à fait imprévisibles sur les images de soi. L'évolution du personnage d'Annie est exemplaire à cet égard. Ainsi, si celle-ci se croyait capable de contrôler le jeu de mémoire qu'elle initie -- c'est elle qui révèle aux psychiatres l'histoire de vie de Thomas et qui pousse ce dernier à se rappeler son passé et à en parler --, elle doit rapidement se rendre à l'évidence que la mémoire commence à se jouer d'elle. En effet, quoique n'ayant pas prévu revenir à l'hôpital et, pour ce faire, prendre un autre jour de congé, elle finit par le faire, car les souvenirs de Thomas qui séjournent dans sa mémoire continuent implacablement à remonter à la surface. Certes, il est plausible, comme elle le mentionne, que les psychiatres lui aient demandé de revenir, mais il s'agit là au pire d'un prétexte et au mieux d'une raison supplémentaire à la motivation première de son retour, à savoir un besoin latent de réconciliation avec son passé. Ce besoin, Annie en nie d'abord l'existence. À la vérité, c'est seulement à la suite de trois nuits blanches, après avoir vu la photo de Thomas 
dans le journal, qu'elle se résout à aller lui rendre visite. Qui plus est, même une fois engagée dans le processus de remémoration, en apparence encore à la faveur de Thomas, Annie refuse d'en admettre la nécessité pour elle-même: «ANNIE : Je devrais pas continuer. [...] Je suis ben, aujourd'hui. Je suis heureuse. J'ai pas besoin de me rappeler des vieilles histoires [...]» (p.44). Toutefois, au cours de la deuxième journée de visite, la parole mémorielle imposera graduellement les règles du jeu : "ANNIE : T'es en train de me descendre avec toi dans le trou de la mémoire où c'est que nos mauvais souvenirs sont tous effacés. Mais. Undelete. Comme sur un ordinateur. Undelete. »(p. 59)

Elle s'y emploie tant et si bien qu'à la fin de la journée, elle parvient à se déployer de toutes ses forces et sous de multiples formes engageant tous les sens :

ANNIE: Tu sais, quand je pense à toi, ça se passe partout en moi. T'es sous ma peau. Dans ma bouche. Dans mon nez. Dans mes oreilles. Dans mes yeux. Pleins de souvenirs endormis [...]. (p. 61)

ANNIE : Où je suis, là? J'ai réveillé tout ce qu'y avait de toi qui dormait en moi. Depuis dix ans, tu dors dans mon corps. C'est peut-être pour ça que j'ai de la misère à dormir depuis dix ans. (p. 62)

Il en découle que l'opiniâtre travail de la mémoire, qui fait ressurgir ce à quoi Annie ne s'attendait pas, l'oblige à réinterpréter son histoire personnelle, plus précisément les dix dernières années de sa vie, qui ne lui ont pas permis, comme elle le croyait ou s'entêtait de le croire, de mettre au diapason passé et présent.

À l'encontre de Thomas, cependant, la réactualisation de la mémoire débouche dans le cas d'Annie sur une 
extériorisation, dans la mesure où, tout en verbalisant ses souvenirs et les états affectifs qui s'y rattachent, elle souhaite partager l'expérience de réminiscence comme la recherche de réconciliation du passé et du présent avec celui qui s'y trouve étroitement impliqué :

ANNIE: Tu sens rien, toi? Rien, hein? Moi, je suis pleine de vieilles sensations de toi. Pis toi, t'es vide. Vide de tout. Même de moi. Vide, câlice! Laisse-moi pas de même. Je peux pas vivre comme ça. Je pourrai pus jamais dormir. T’es réveillé en moi. Dis quelque chose! (p. 62)

ANNIE : J'en ai assez dit. Là, c'est à ton tour, Thomas. J'ai parti le bal. À toi maintenant. Je peux pas tout dire. Je suis pas ici pour une confession. C'est une conversation que je veux. Thomas? (p.63)

Cet appel à un dialogue restera, nous l'avons déjà noté, sans réponse de la part de Thomas, état de fait qui paraît moins relever d'une incapacité mnémonique ou mentale que d'un choix personnel. En effet, si les deux justifications de la condition de Thomas avancées au début de la pièce, soit l'amnésie et la folie (la déraison), ne manquent pas d'atteindre l'intégrité d'un sujet, force est de constater que c'est principalement son refus d'entretenir un contact réel avec autrui qui engendre sa difficile situation. Le confirment aussi bien Annie-2 ${ }_{12}$ que Marthe ${ }^{13}$, et Annie s'en rendra également compte ${ }^{14}$. Par conséquent, ayant remarqué que les souvenirs et les sentiments qu'elle exprime devant Thomas ne semblent lui

\footnotetext{
12 «ANNIE-2 : [...] Il veut pas reconnaître personne. C'est pour ça qu'il est ici [l'hôpital psychiatrique]. Pour pas se rappeler.» (p. 30; nous soulignons) 13 «MARTHE : [...] Parce qu'il fait semblant. Semblant de pas parler, semblant de pas se souvenir. Il veut se mentir à lui-même [...]. » (p. 51; nous soulignons) 14 «ANNIE : Je peux pas croire que tu te rappelles de rien. Tes yeux mentent pas [...]. » (p. 59) Nous explorerons plus en profondeur l'importance de cette observation dans la prochaine section de l'étude.
} 
faire ni chaud ni froid du point de vue d'une réaction réellement verbale, Annie s'efforce, de manière physique, corporelle, de ranimer les organes de ses sens et de la parole. D'abord, elle le gifle. Ensuite, « [e]lle lui saisit le menton » (p. 68) ${ }^{15}$, voulant qu'il la regarde dans les yeux; "[e]lle lui met la main sur la bouche» (ibid.), lui enjoignant de parler; "[e]lle lui tire les oreilles» (ibid.), en lui demandant s'il l'entend; «[e]lle le secoue violemment » (ibid.), en criant son nom, cherchant à susciter une quelconque réaction. Une fois calmée, Annie fait même montre de la profonde affection qu'elle éprouve toujours pour celui qu'elle n'a pas vu pendant une décennie en l'embrassant. Bien que toutes ces tentatives d'Annie pour ouvrir les voies de la communication entre elle et Thomas, et pour faire du même coup sortir ce dernier de son mutisme, se soldent par un échec, elle en viendra finalement à assumer pleinement son passé et à s'en délivrer.

La réconciliation d'Annie avec elle-même est rendue possible grâce au concours d'un autre personnage embrouillé dans son histoire de vie comme dans celle de Thomas : Ève, leur enfant, qu'Annie avait, après tout, décidé de garder. L'influence de ce personnage au nom à valeur fort symbolique apparaît nettement à l'occasion du troisième jour de visite :

ANNIE : Hier, quand je suis partie, je voulais pas revenir. J'étais crevée. Mais. Ça a dû me faire du bien dans le fond parce que j'ai bien dormi cette nuit. Tellement bien que j'ai oublié jusqu'à mon nom. En me réveillant, ce matin, ça m'a pris un temps avant de reconnaître où j'étais, qui j'étais. C'est la voix d'Ève qui m’a ramenée à moi. (p. 88)

15 Ce geste d'Annie ainsi que les trois suivants sont indiqués dans les didascalies, d'où l'italique dans le texte cité. 
Pour Annie, la rencontre avec Thomas, quoique ravivant d'emblée de vieux souvenirs torturants, s'avère donc cathartique en ce qu'elle débouche, avec l'appui d'Ève, sur une espèce de renaissance. Non pas qu'Annie fasse table rase de son passé, tant s'en faut; elle l'endosse plutôt et y met de l'ordre. Autrement dit, après avoir provisoirement perdu connaissance de son premier trait identificatoire («j'ai oublié jusqu'à mon nom ») ainsi que de son image de soi au total (« où j'étais, qui j'étais »), Annie procède à une reconfiguration, plus précisément à une refiguration, de son histoire de vie en tenant compte non seulement des échanges récents avec Thomas, mais aussi et surtout de l'importance qu'a sa fille dans sa vie. Tout bien considéré, c'est cette refiguration de son histoire de vie qui lui permet de se décharger du fardeau accablant que son passé représentait jusqu'alors à ses yeux et de se modeler une nouvelle image de soi où passé et présent s'harmonisent.

Désormais consciente que «l'oubli ça comble pas le vide [sic] » (p. 92) et ne voulant pas occulter les origines de sa fille, Annie décide de l'emmener avec elle le troisième et dernier jour de sa visite à l'hôpital afin de la présenter à Thomas. Bien qu'aucun échange verbal n'ait lieu entre père et fille, il n'en demeure pas moins que celle-ci désire un contact avec lui. De fait, en plus d'offrir à son père un dessin représentant ellemême, sa mère et l'immeuble où elles habitent, et indiquant même leur adresse et leur numéro de téléphone, Ève tenait à ce que sa mère offre à Thomas un cadeau. Aussi, contrairement à ses propres convictions ${ }^{16}$, Annie présente-t-elle à celui-ci, dans un geste quasiment apotropaïque, un nouveau sac d'épicerie

16 «ANNIE : [...] D’habitude, je donne pas aux quêteux. [...] Mais t'es le père de ma fille. [...] Fa que. Je fais une exception [...] [sic]. » (p. 90-91) 
contenant lui aussi trois objets: un «sac de biscuits», des « mitaines » et une " photo [d'école] d'Ève» (p. 91). Ce dernier objet est hautement significatif, car c'est en le contemplant après le départ de ses deux visiteuses que Thomas, maintenant seul dans la salle d'observation et en attente de sa libération, prononce son premier et unique mot, «Ève» (p. 92), réplique finale de la pièce.

Comment interpréter ce dénouement à la lumière du kaléidoscope de fragments narratifs et de la nécessaire interdépendance des images de soi en devenir qui est ainsi mise en valeur? Fin euphorique? Fin dysphorique? Si l'une et l'autre interprétations se valent ${ }^{17}$, il semble bien que la première prime la seconde. En ce sens, compte tenu de l'effet bénéfique de la naissance d'Ève sur le parcours d'Annie, effet que Marthe avait, soit dit en passant, prédit à cette dernière dix ans plus tôt (p. 76), il y a fort à parier que la fille peut agir pareillement sur son père. Après tout, la présence d'Ève le dernier jour de visite à l'hôpital ne permet-elle pas de faire taire les trois voix fantomatiques qui obsèdent Thomas? Par ailleurs, le fait que ce dernier prononce le nom de sa fille n'ouvre-t-il pas une première brèche dans son mutisme? N'est-ce pas par là qu'Ève l'aide à revenir à lui-même, tout comme elle l'a déjà fait pour sa mère? Enfin, que "[I]es bruits de la ville envahissent l'espace » (p. 92) ${ }^{18}$ à la toute fin de la pièce, cela ne signifie-t-il pas que Thomas, quoi qu'il en soit de l'incertitude quant à son cheminement ultérieur, retourne à la réalité et qu'il se met finalement à composer avec sa situation actuelle? C'est du moins ce que signalent, en filigrane, les trois nouveaux objets

17 Voir notamment Dominique Lafon (1999) et Jane Moss (2000).

18 Il s'agit d'une indication en didascalie, d'où l'italique dans le texte cité. 
dans son sac, branchés qu'ils sont sur le présent. Il s'ensuit que la fin de la pièce laisse malgré tout entrevoir une lueur d'espoir pour ce qui est du destin des trois personnages réels mis en scène.

\section{Réflexivités : entre auto-images et hétéro-images}

À l'encontre de ce que pourrait suggérer le titre de l'œuvre, le principe de réflexivité, au sens large de mouvement du sujet revenant sur lui-même ${ }^{19}$, est très net du début à la fin de L'Homme effacé. La scène qui clôt la pièce se révèle certainement d'un grand intérêt à cet égard, dans la mesure même où elle marque un retour en force de l'image, incarnée à la fois par le dessin d'Ève et par sa photo. Retour en force puisque, outre que nous nous attachons depuis le début de cette étude à cerner les modalités de construction des images de soi, ce n'est pas, comme on le verra, la première fois que l'apparition d'une image infléchit le comportement des personnages de L'Homme effacé.

La mise en valeur de l'image dans la pièce est d'autant plus importante qu'elle dénote la place primordiale de l'autre dans les configurations identitaires, non seulement à titre de «co-personnage», oserait-on dire, dans l'histoire d'une vie, mais encore à titre de lieu de médiation nécessaire à la réflexivité constitutive de toute formation d'image de soi. De fait, le façonnement des images de soi est conditionné autant,

\footnotetext{
${ }^{19}$ Sur la place capitale de l'activité réflexive dans la construction identitaire, se référer notamment à Anthony Giddens (1991) et à Jean-Claude Kaufmann (2007a, 2007b).
} 
sinon davantage, par les " hétéro-images » - la perception de soi par l'autre, soit-il individuel ou collectif 20 - que par les " auto-images » - la perception de soi par soi-même ${ }^{21}$. Comme le précise Charles Taylor dans son étude sur le «multiculturalisme » et les « politiques de reconnaissance»,

[...] our identity is partly shaped by recognition or its absence, often by the misrecognition of others, and so a person or group of people can suffer real damage, real distortion, if the people or society around them mirror back to them a confining or demeaning or contemptible picture of themselves. Nonrecognition or misrecognition can inflict harm, can be a form of oppression, imprisoning someone in a false, distorted, and reduced mode of being. (p. 25)

De même, Jean-Claude Kaufmann souligne, dans L'Invention de soi, le caractère multiple des images de soi. Celles-ci

doivent être analysées au pluriel, car elles sont de types différents (mais circulent de façon très fluide entre ces catégories). Il y a les images sociales, reflets de la structure. De

${ }^{20} \mathrm{~L}$ '« autre collectif» renvoie dans ce contexte aussi bien à différents genres de communauté (tels les ensembles nationaux, ethniques ou religieux) qu'à diverses structures institutionnelles lato sensu (tels les milieux familiaux et professionnels).

21 Les termes d'auto-image et d'hétéro-image proviennent du domaine de l'imagologie. Voir par exemple Joep Leerssen (1997) et Menno Spiering (1992). Cependant, nous nous permettons ici de modifier dans une certaine mesure leur signification et leur champ d'application respectifs en les rapportant à la fois aux collectivités (auxquelles on les associe ordinairement) et aux individus, et en dotant le terme d'hétéro-image d'une réflexivité intrinsèque. C'est que, d'habitude, on ne lui reconnaît une valeur réfléchie qu'après coup en quelque sorte. En effet, l'hétéro-image désigne au sens strict du terme l'image de l'étranger, c'est-à-dire l'image que le soi se fait de l'autre, par opposition à l'image du propre, à l'auto-image. Sous cet angle, ce n'est qu'en considérant les influences mutuelles entre des ensembles distincts d'auto-images et d'hétéro-images ressortissant chacun à un sujet spécifique qu'il devient possible de prendre en compte la dimension réflexive des formations identitaires. 
plus en plus individualisantes, car formées autour de positions très spécifiques. De plus en plus contradictoires et multiples aussi, reflets bigarrés des rôles disparates pouvant être tenus par un même individu. Il y a les images de soi que se forme autrui. Autrui est ce drôle de personnage, qui, justement, ramène toujours votre identité [...] à une simple image. Il vous réduit à deux ou trois critères, et rêve de vous fixer à jamais [...]. Et puis il y a vous-même, qui essayez de tordre dans le bon sens la réalité trop grise, en vous mettant en scène dans un " petit cinéma » secret et très visuel, qui n'est rien d'autre qu'une expérimentation imaginaire d'identités possibles, par des prises de rôles virtuelles [...]. (p. 69-70; nous soulignons)

Passages qui font clairement ressortir non seulement la nature plurielle et dynamique de l'image de soi ${ }^{22}$, mais encore le rôle crucial que jouent la réflexivité et l'intervention d'autrui dans les constantes modulations et transformations de cette image. Il est évident que L'Homme effacé thématise ce rôle de médiateur de l'autre par les télescopages de perspectives sur les bribes de vécu des différents personnages, perspectives qui prennent tout leur sens dans leurs éclairages mutuels, dans leur réciprocité.

22 Cette conception de l'image de soi en tant que plurielle et dynamique par essence n'est pas celle de Jean-Claude Kaufmann. "L'image, affirme-t-il, est [...] un moment obligé dans la construction sociale de l'individu [...]. Elle n'est cependant pas tout. Elle n'est précisément qu'un moment, un moment bref. Elle trompe par omission. En créant l'illusion de tout dire par son instantanéité totalisante.» (2007b, p. 70) Cela dit, les observations du sociologue sur les «types différents » d'image de soi restent très éclairantes pour notre propos. Surtout qu'à y regarder de près, il s'en détache, en sourdine, une représentation de l'image de soi qui participe d'un certain dynamisme (les images « de types différents » « circulent de façon très fluide entre ces catégories »). En outre, la question reste ouverte de savoir si, quel que soit le terme employé (identité, image de soi), il y aurait jamais moyen d'esquiver la nature illusoire et non totalisante de la perception d'un sujet, celle-ci étant irrémédiablement ancrée dans un hic et nunc. 
Dans ce contexte, il convient de souligner la place centrale du regard et de la réflexivité qui s'y rattache dans le déploiement de l'intrigue. À cet effet, notons tout d'abord que l'épisode tragique de Thomas dans la rue Yonge comme l'internement qui en résulte sont provoqués par le regard d'une jeune fille, à savoir Ève, alors qu'elle dépose de la monnaie dans la main d'un sans-abri, qui s'avère celle de Thomas: «MARTHE : Thomas. Les yeux que t'as vus, y a une semaine, sur le coin de Yonge Street. Les yeux qui t'ont tant fait peur. [...] Ces yeux-là sont liés à toute ton histoire avec celle qui vient de partir [Annie] » (p. 51).

Ayant reconnu dans les yeux de la fillette ses yeux à lui ${ }^{23}$, Thomas tente, nous l'avons vu, de trouver dans le silence absolu et l'oubli forcé des palliatifs au trouble qu'il éprouve à la suite de cette rencontre ${ }^{24}$. Si, dans cette perspective, le regard constitue l'élément déclencheur de l'intrigue, il joue un rôle tout aussi décisif dans son déroulement, ainsi qu'en témoignent les interactions entre Annie et Thomas. En ce sens, c'est la photo de celui-ci, plus précisément ses yeux, qui confirment à Annie que l'annonce de journal publiée par les autorités traite véritablement de lui :

ANNIE : J'ai failli pas venir. Je me suis dit que ça pouvait pas être lui. [...] Mais. C'était ben toi sur la photo dans le journal. Les mêmes yeux. J'ai vu que c'était toi [...]. (p. 22)

\footnotetext{
23 Voir aussi L'Homme effacé, p. 29.

24 Quoique l'on puisse tenir la première réaction de Thomas à cette rencontre pour involontaire, sa volonté est pour ainsi dire restaurée une fois le choc initial passé. Comme l'attestent Marthe, Annie-2 et Annie dans les extraits déjà cités, Thomas, une fois rendu à l'hôpital, détient la capacité de décider de la manière de composer avec sa situation, et il choisit de rompre le contact avec l'autre.
} 
ANNIE : C'est à cause de la photo dans le journal. À cause de tes yeux. [...] Dans tes yeux, je me suis vue. Comme si j'étais dans tes yeux. Trois nuits que je dors pas. Fa que je me suis finalement décidée à venir voir si c'était bel et bien toi [...]. (p. 23)

Si le regard de Thomas amorce alors la descente d'Annie dans les tumultueux flots de la parole mémorielle, il lui servira également de guide dans cette descente : "Je peux pas croire que tu te rappelles de rien. Tes yeux mentent pas [...]» (p. 59), déclare-t-elle à Thomas le deuxième jour de sa visite à l'hôpital. Ainsi, ce sont encore les yeux du héros qui se montrent révélateurs, cette fois-ci en convaincant Annie qu'il ne souffre pas d'amnésie, mais opte volontairement pour le silence. Cette prise de conscience d'Annie dirigera la suite de ses interactions avec le père de sa fille, y compris les efforts déjà mentionnés pour l'amener à communiquer avec elle. Il ne fait donc pas de doute qu'Annie reconnaît d'entrée de jeu l'importance des échanges mutuels et de la réflexivité, dont participe le regard de l'autre, dans la formation de son image de soi.

Il en va tout autrement pour Thomas. Tandis que se voir dans les yeux d'autrui pousse Annie à engager un échange avec lui, chez Thomas, cela débouche sur un repli sur soi :

MARTHE : Quelqu'un a planté ses yeux dans tes yeux. Sur un coin de Yonge Street. [...] C'est à cause de ces yeux-là que tu refuses de parler. [...] C'est pour fuir ces yeux-là que t'es allé marcher sur la ligne jaune [ligne qui départage les deux sens de la circulation]. [...] Avec l'espoir de te faire frapper. (p. 68)

Après avoir aperçu les yeux d'Ève, Thomas s'efforce donc aussitôt de rompre toute relation avec autrui, de répudier toute possibilité de réciprocité. Cette réaction ne saurait avoir qu'un impact négatif sur son image de soi, car un manque de réciprocité et de réflexivité conduit tout droit à la sclérose et la perpétuation 
du Même. L'attestent autant les redites presque obsessionnelles du récit de vie de Pite (p. 12,19) que le genre de vie que Thomas a menée pendant dix ans dans les rues de la métropole torontoise :

MARTHE : [...] Dans ton ventre, la même faim. Dans les os, le même froid. Toutes tes misères pis tous tes malheurs. Replié cent fois, mille fois dans ton histoire. Cent fois, mille fois, t'as parlé tout seul. Cent fois, mille fois, t'as suivi des filles qui ressemblaient à Annie. Cent fois, mille fois. (p. 68-69)

En reniant de la sorte les rapports de réciprocité et de réflexivité, Thomas se barricade en fin de compte dans une sorte d'existence monotone, circulaire, équivalant à rien de moins que « a false, distorted, and reduced mode of being », pour reprendre les mots de Charles Taylor. N'est-ce pas, précisément, une manière d'être réduite que traduit l'effacement évoqué par le titre de la pièce? Que le héros en soit un homme effacé renvoie dans cette optique moins à une perte de mémoire-- les fantômes qui s'agitent dans sa tête signalent plutôt qu'il a un trop-plein de mémoire -- ou à une perte de raison qu'au manque de réflexivité assumée par le personnage sur le plan de ces interactions effectives avec le monde qui l'entoure. Au final, c'est cette rebuffade de la réflexivité qui empêche Thomas de faire "s'emboîte[r] comme il faut», de manière convenable, ce "gros casse-tête» (p.68) qu'est l'histoire de sa vie avec autrui et d'adopter en conséquence une image de soi harmonieuse.

\section{Conclusion}

Au terme de cette traversée dans les aires brumeuses de l'identité, de l'altérité et de la mémoire, traversée éclairée pour l’occasion par L'Homme effacé de Michel Ouellette, on peut constater que les rapports du soi à l'autre, loin d'être réductibles à une privation, 
relèvent au contraire d'une relation foncièrement interdépendante, et d'une intersignifiance fondamentale. Car il n'est pas de sens de soi sans sens de l'autre. Cette intersignifiance est portée nettement au jour par l'implication d'autrui dans diverses expériences et reconfigurations mémorielles de même que dans les reformulations du sens de soi qui en émanent. Il se trouve, en effet, que l'autre, quelle que soit la nature de son implication, joue tant bien que mal un rôle primordial dans le modelage des images de soi, images qui ne sauraient se comprendre et se communiquer que par voie/voix narrative. En d'autres termes, compte tenu de l'interdépendance et de l'enchevêtrement fondamentaux de l'existence humaine, autrui participe de façons multiples et diversifiées à la mise en intrigue comme à l'interprétation de l'histoire de vie d'un sujet.

À la lumière de ces observations, on pourrait affirmer en somme que l'image de soi, plurielle et narrativement comprise et articulée, se forge par le biais d'une perpétuelle oscillation entre auto-images et hétéro-images, peu importe la direction de leur dilatation dans le temps (rétrospection ou prospection) et l'ampleur de cette dilatation, et peu importe les motivations et les orientations qui les déterminent. Or, pour que soient possibles cette oscillation constitutive des façonnements identitaires et le dynamisme régénérateur qui lui est inhérent, il faut qu'il y ait un écart, un décalage, une distance, bref une différence entre le soi et l'autre. Ce n'est qu'en reconnaissant cette différence comme positive ou constructive, au lieu de l'envisager sous une lumière négative ou destructive, que l'on peut décentrer l'identité du soi et désaliéner l'altérité de l'autre, et accorder dès lors le présent avec le passé et l'avenir. 


\section{Bibliographie}

BAKHTINE, Mikhaïl (1979), Estetika slovesnogo tvorchestva [Esthétique de la création verbale], Moscou, Iskusstvo.

FouCAulT, Michel (1972 [1961]), Histoire de la folie à l'âge classique suivi de Mon corps, ce papier, ce feu et La folie, l'absence d'œuvre, nouv. éd., Paris, Gallimard, coll. «Bibliothèque des histoires ».

GIDDENS, Anthony (1991), Modernity and Self-Identity. Self and Society in the Late Modern Age, Stanford, Stanford University Press.

KARSKENS, Machiel (1991), «Alterity as Defect: On the Logic of the Mechanism of Exclusion ", dans Raymond Corbey et Joep Leerssen (dir.), Alterity, Identity, Image : Selves and Others in Society and Scholarship, Amsterdam, Rodopi, coll. "Amsterdam Studies on Cultural Identity », p. 75-90.

Kaufmann, Jean-Claude (2007a [2001]), Ego. Pour une sociologie de l'individu, Paris, Hachette Littératures, coll. « Pluriel ».

- (2007b [2004]), L'Invention de soi. Une théorie de l'identité, Paris, Hachette Littératures, coll. « Pluriel ».

KristeVA, Julia (2004 [1988]), Étrangers à nous-mêmes, Paris, Gallimard, coll. « Folio/Essais ».

LAFON, Dominique (1999), «L'Homme effacé : pièce de théâtre de Michel Ouellette », Francophonies d'Amérique, no 9, p. 213216.

LALANDE, André (2006 [1926]), Vocabulaire technique et critique de la philosophie, 2e éd. "Quadrige», Paris, Presses universitaires de France, coll. «Quadrige/Dicos poche». 
LEERSSEN, Joep (1997 [1986]), Mere Irish and Fíor-Ghael: Studies in the Idea of Irish Nationality, its Development and Literary Expression prior to the Nineteenth Century, 2e éd., Notre Dame, University of Notre Dame Press, coll. "Critical Conditions : Field Day Monographs ».

LOCKE, John (1788), "Of Identity and Diversity», [Chap. XXVII], An Essay Concerning Human Understanding. In Four Books, 18e éd., 2 vol., Londres, [s. é.], p. 344-390.

Memmi, Albert (1985 [1957]), Portrait du colonisé précédé de Portrait $d u$ colonisateur, Paris, Gallimard, coll. «Folio/Actuel».

Moss, Jane (2000), "Le théâtre franco-ontarien: Dramatic Spectacles of Linguistic Otherness », University of Toronto Quarterly, vol. 69, n² 2, automne, p. 587-614.

OuElLET, Pierre (2003), Le Sens de l'autre. Éthique et esthétique, Montréal, Liber.

OUELLETTE, Michel (1997), L'Homme effacé, Ottawa, Le Nordir.

— (1999), Tombeaux, Vanier, L'Interligne, coll. «Vertiges ».

- (2007), "Parcours sous influence», Theatre Research in Canada/Recherches théâtrales au Canada, vol. 28, no 1 , printemps, p. 54-68.

PERrY, John (dir.) (2008 [1975]), Personal Identity, 2e éd., Berkeley/Los Angeles/Londres, University of California Press.

RENOUVIER, Charles (1930), " De la relation comme catégorie ", dans Les Derniers Entretiens, Louis Prat (éd.), Paris, J. Vrin, coll. « Bibliothèque des textes philosophiques ».

RICOEUR, Paul (1991 [1985]), Temps et récit. Tome III. Le Temps raconté, Paris, Seuil, coll. « Points Essais ».

- (1996 [1990]), Soi-même comme un autre, Paris, Seuil, coll. «Points Essais ». 
- (2003 [2000]), La mémoire, l'histoire et l'oubli, Paris, Seuil, coll. « Points Essais ».

ScHAPP, Wilhelm (1976), In Geschichten verstrickt. Zum Sein von Mensch und Ding, Wiesbaden, B. Heymann.

SPIERING, Menno (1992), Englishness. Foreigners and Images of the National Identity in Postwar Literature, Amsterdam/ Atlanta, Rodopi, coll. « Studia Imagologica ».

TAYLOR, Charles (1994 [1992]), «The Politics of Recognition ", Multiculturalism : Examining the Politics of Recognition, éd. aug., Amy Gutmann (éd.), Princeton, Princeton University Press.

Todorov, Tzvetan (1981), Mikhaïl Bakhtine: le principe dialogique suivi de Écrits du Cercle de Bakhtine, Paris, Seuil, coll. «Poétique».

- (1997 [1991]), Les Morales de l'histoire, Paris, Hachette Littératures, coll. « Pluriel ».

- (2001 [1989]), Nous et les autres. La réflexion française sur la diversité humaine, 2e éd., Paris, Seuil, coll. « Points Essais ».

\section{Résumé}

Cette étude explore les relations intersignifiantes entre le soi et l'autre sous l'optique du travail de la mémoire inhérent au devenir de tout être. Son objectif est de sonder, à travers une réflexion sur L'Homme effacé de Michel Ouellette, la place qu'occupe l'autre dans les reconfigurations mémorielles qui influent sur les continuités et les ruptures identitaires. Elle vise notamment à mettre en lumière que les images de soi, en tant que constructions narrativement comprises et articulées, se 
façonnent à même le lieu mouvant de différence entre les perceptions de soi par soi-même et les perceptions de soi par l'autre, quelles qu'en soient les déterminations temporelles et motivationnelles.

\begin{abstract}
This study explores the inter-significant relations between the self and the other by looking at the workings of memory in the constant evolution of one's being. Its aim is to sound out, through a reflection on Michel Ouellette's L'Homme effacé, the role of the other in the reconfigurations of memory that affect the continuities and discontinuities of identity. In particular, it intends to bring to light that self images, as narratively understood and articulated constructs, take shape within the shifting locus of difference between perceptions of the self by oneself and perceptions of the self by the other, whatever their temporal and motivational determinations.
\end{abstract}

